Pirineos. Revista de Ecología de Montaña

Vol. 175

Jaca, Enero-Diciembre, 2020, e054

ISSN-1: 0373-2568

https://doi.org/10.3989/pirineos.2020.175004

\title{
DETERMINACIÓN DE LA DISTRIBUCIÓN ACTUAL Y POTENCIAL DEL ÁGUILA REAL (Aquila chrysaetos) EN EL SECTOR OCCIDENTAL DE ÁLAVA (PAÍS VASCO)
}

\section{Determination of the current and potential distribution of the Golden Eagle (Aquila chrysaetos) in the occidental sector of Alava (Basque Country)}

\author{
Pedro José Lozano Valencia* y Peio Murua Alzola \\ Universidad del País Vasco/Euskal-Herriko Unibertsitatea. \\ Identificador ORCID de los autores y e.mail \\ Pedro José Lozano Valencia: http://orcid.org/0000-0002-1345-5704. E.mail: pedrojose.lozano@ehu.eus \\ Peio Murua Alzola: https://orcid.org/0000-0002-4485-445X. E.mail: pmurua001@ikasle.ehu.eus \\ *Autor corresponsal
}

Recibido: 02-04-2020. Aceptado: 01-06-2020. Fecha de publicación on-line: 08/09/2020

Citation / Cómo citar este artículo: Lozano Valencia, P. J., Murua Alzola, P. (2020). Determinación de la distribución actual y potencial del águila real (Aquila chrysaetos) en el sector occidental de Álava (País Vasco). Pirineos, 175, e054. https://doi.org/10.3989/pirineos.2020.175004

RESUMEN: El águila real (Aquila chrysaetos) es una de las aves más grandes y emblemáticas de la península Ibérica. En dicho territorio se ha llegado a calcular la existencia de 1.500 parejas, población muy reducida y, por ello, catalogada bajo diversas figuras de protección. El objetivo del trabajo es realizar una estimación de sus contingentes poblacionales dentro del sector occidental de Álava. Estos nuevos datos serán cotejados con otros inventarios anteriores. A través de un complejo y contrastado proceso metodológico derivado de transectos regulares, realizados durante dos años y acompañados de visitas a lugares sensibles junto a estaciones de vigilancia, se ha realizado la estimación poblacional a la vez que se han estudiado otras variables como la dieta, la ubicación de los nidos y las parejas, los movimientos de dispersión y las posibilidades de distribución potencial dentro de este ámbito territorial. Los resultados hablan de densidades relativamente altas y de una distribución en aquellos sectores con gran cantidad de presas, poco impactados por el ser humano, con poca presión antrópica y con un paisaje en el que alternan bosques bien conservados y paisaje de campiña y pastos. Seis han sido los territorios y parejas confirmadas mientras que podría haber otras dos parejas más.

PALABRAS CLAVE: Aquila chrysaetos; orografía; usos del suelo; parejas reproductoras; Álava occidental.

ABSTRACT: The golden eagle (Aquila chrysaetos) is one of the largest and most emblematic birds of the Iberian Peninsula. In this territory the existence of 1,500 couples has been calculated, a very small population and 
therefore listed under various management categories. The objective of the work is to estimate its population quotas within the western sector of Alava. This new data will be cross-checked against previous inventories. Through a complex and proven methodological process derived from regular transects carried out over two years and accompanied by visits to sensitive places next to surveillance stations, the population estimate has been made while studying other variables such as diet, nest and couples location, dispersal movements and potential distribution possibilities within this territorial area. The results speak of relatively high densities and a distribution in those sectors with a large amount of prey, little impacted by humans, with little anthropic pressure and a landscape a well-preserved forests landscape in which countryside and pasture coexist. Six have been the territories and couples confirmed while two are in doubt.

KEY WORDS: Aquila chrysaetos; orography; land uses; breeding pairs; western Alava.

\section{Introducción}

El águila real (Aquila chrysaetos L., 1758) es una de las aves más grandes y emblemáticas del hemisferio norte. Pertenece a la familia Accipitridae. Su distribución actual engloba diversas regiones como Eurasia, Norteamérica y norte de África. No obstante, dentro de esta gran distribución hay que reseñar que está ausente de países como Francia, Alemania, Holanda, Bélgica o Polonia (Arroyo, 2003). A su vez, dentro de la especie se diferencian subespecies como la que se encuentra en el este y sector central de Europa, así como el oeste de Rusia (Aquila chrysaetos chrysaetos), en Asia central (Aquila chrysaetos daphanea), en Japón y Corea (Aquila chrysaetos japonica), al este de Siberia (Aquila chrysaetos kamtschatica), en Norteamérica, hasta el norte de México, (Aquila chrysaetos canadensis) y, por fin, en la península ibérica y el norte de África (Aquila chrysaetos homeyeri). Habría que destacar que existió una séptima subespecie desaparecida en el Holoceno (Aquila chrysaetos simurgh) (Ibor, 2014).

La población global de la especie se calcula entre 170.000 y 200.000 individuos aunque no se puede saber con exactitud (Arroyo, 2004). En Europa se estima que pueden existir entre 6.600 y 12.000 parejas reproductoras lo que nos acercaría a un número total de individuos de entre 13.200 y 24.000 (Ibor, 2014). En la península ibérica diversos autores han llegado a calcular la existencia de 1.500 parejas, de manera que muestra unos contingentes poblacionales relativamente reducidos $\mathrm{y}$, por ello, se encuentra catalogada bajo diversas figuras de protección (Arroyo, 2004; Del Moral, 2009; Ibor, 2014). Según la UICN se encuentra catalogada como de preocupación menor. Sin embargo, en el territorio estatal la especie aparece catalogada en el atlas y libro rojo de las aves bajo la categoría de casi amenazada, mientras en la Comunidad Autónoma del País Vasco (CAPV) se encuentra en la categoría de vulnerable. En cualquier caso, en España la especie se encuentra protegida dentro de la lista de especies silvestres en régimen de protección especial, de manera que no puede ser cazada así como recibir molestias o cualquier otro impacto negativo (Arroyo, 2003 y 2004).
Dentro de la península ibérica su distribución es realmente irregular de manera que, en general, ocupa los espacios montanos y las grandes cordilleras: Cordillera Cantábrica, Pirineos, Cordillera Ibérica, Sistema Central, Cordilleras Béticas, Montes de Toledo y Sierra Morena. Aunque en estos enclaves alcanza sus mayores densidades, también ocupa otros sectores no montañosos como el Valle del Ebro o el Bierzo. Falta de amplios sectores de las dos submesetas y de la mayor parte de las costas atlánticas, cantábricas y mediterráneas (Arroyo, 2004).

Los ejemplares pueden alcanzar un peso que oscila entre 3 y $6 \mathrm{~kg}$ y que depende del sexo, estado de desarrollo y alimentación. La hembra siempre es más grande que el macho (Figura 1). Cuenta con un plumaje, en general, marrón oscuro y con tonos amarillentos tanto en las alas como a la altura del cuello. Al volar, por la parte de abajo, presenta un plumaje de color más grisáceo con tonos blancos derivados de la existencia de manchas de este color en la base de ciertas plumas. Cuando son jóvenes y se han emancipado del nido su plumaje es más oscuro, prácticamente negro, mientras que cuando nacen lo hacen cubiertos de un plumón blanco que va desapareciendo a medida que va creciendo. Sus patas muestran tarsos amarillos y culminan en 4 garras muy afiladas y largas de color negro, tres en posición delantera y otra trasera que llega a medir hasta 7 $\mathrm{cm}$. El pico presenta un color claramente amarillo a la altura de las narinas mientras que hacia la punta se oscurece siendo totalmente negro, afilado y muy potente. Con el pico puede agujerear y desgarrar cualquier tipo de carne o presa. Los ojos son negros, grandes y se encuentran en una posición frontal de manera que cuenta con visión estereoscópica. Su envergadura de pico a cola puede oscilar entre 76 y $103 \mathrm{~cm}$ dependiendo del sexo y edad. Igualmente, de ala a ala puede oscilar entre 180 y $230 \mathrm{~cm}$ (Arroyo, 2003).

Aunque al volar alcanza altitudes relativamente elevadas, en caso de avistamiento de presa, es capaz de plegar las alas y descender a una velocidad relativamente alta, muy parecida a la que muestra el halcón peregrino (Falco peregrinus) de manera que puede llegar a alcanzar los $250 \mathrm{~km} / \mathrm{h}$, situándose entre los animales más veloces del mundo (Díaz et al., 1983). Por si eso fuera poco, con el 


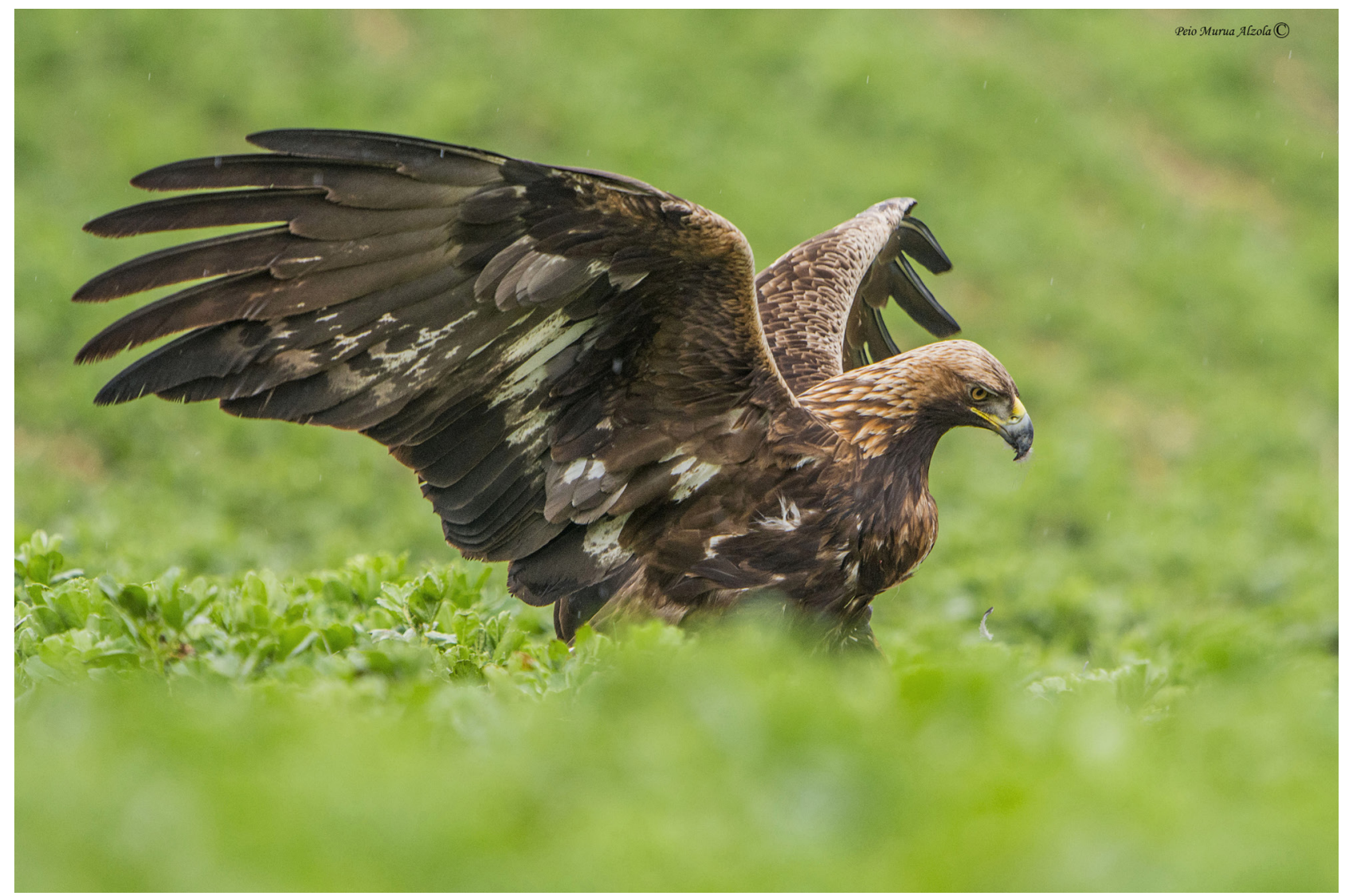

Figura 1: Ejemplar de Águila real, en este caso una hembra adulta con plumaje perfectamente desarrollado. Fotografía de los autores.

Figure 1: Golden Eagle specimen, in this case an adult female with perfectly developed plumage. Photograph of the authors.

pico asesta potentes picotazos en órganos y zonas críticas para sus presas, de manera que puede dar muerte relativamente rápido incluso a presas tan grandes como el zorro (Vulpes vulpes), el tejón (Meles meles) o el corzo ( $\mathrm{Ca}$ preolus capreolus) (Bautista et al., 2016).

$\mathrm{Su}$ dieta se basa en la captura de diferentes presas como el conejo (Oryctolagus cuniculus), perdiz roja (Alectoris rufa), paloma torcaz (Columba palumbus) y bravía (Columba livia), reptiles como culebras y lagarto ocelado (Lacerta lepida) e incluso mamíferos como la liebre (Lepus sp.), el zorro (Vulpes vulpes) o el corzo (Capreolus capreolus) (Bautista et al., 2016). Esta dieta, junto a su biología, ecología e incluso etología, hace de este ave una de las más escasas, no sólo de la península ibérica, sino a escala mundial. Su dinámica poblacional se fue reduciendo a partir de los años 60 del pasado siglo como consecuencia de cuestiones como la disminución de determinadas poblaciones de presas como el conejo, el abandono agrario, la caza indiscriminada derivada de la ley de alimañas de 1953 y la utilización de diferentes venenos para diezmar a las entonces denominadas como alimañas (Fernández \& Purroy, 1990).
En lo que respecta a sus pautas reproductoras, la especie inicia la época de cortejo y celo hacia finales de enero y durante todo febrero, cuando comienza a dar los primeros vuelos nupciales.

El nido comienza a instalarse una vez que la relación de la pareja se encuentra perfectamente asentada. Este se construye en la repisa de un abrigo rocoso, saliente, cueva, etc. dentro de cortados rocosos de cierta entidad. Es cierto que, en otras partes de Europa e incluso de la península ibérica, a falta de cortados o abrigos rocosos, hay parejas que pueden llegar a instalar sus nidos en árboles de cierto porte (Díaz et al., 1983). También han sido detectadas sendas ubicaciones en Guadalajara y Badajoz, donde la puesta del nido y la cría de los polluelos se realizaron sobre el suelo (Arroyo et al, 1990). En otros casos, más anecdóticos, la instalación del nido se llevó a cabo en un poste de alta tensión (Soria) e incluso, en otro caso, sobre el tejado de una cabaña rústica de montaña (Arroyo, 2000). Para la construcción del nido ambos individuos aportan materiales vegetales como ramas, ramitas, hojarasca, etc. El nido, por lo menos el primer año, no es excesivamente grande ni amplio si tenemos en cuenta la envergadura y peso del taxón, sin 
embargo, si la cría se ha realizado con éxito, es muy frecuente que las parejas vuelvan a utilizar el mismo nido y lo vayan ampliando con nuevos aportes, de manera que la estructura, con el tiempo, puede llegar a alcanzar una gran envergadura, incluso de más de tres metros de diámetro (Arroyo, 2000).

El periodo de incubación se puede prolongar desde finales de febrero hasta principios de abril. Lo más normal es que el periodo se prologue a lo largo del mes de marzo. Dicha incubación dura 43 días y en ella participa mayoritariamente la hembra que no abandonará el nido incluso hasta después de que los huevos hayan eclosionado y cuenten con unos días. Collopy (1984) determinó que el $84 \%$ del tiempo de incubación corre a cargo de la hembra y concluyó que esto puede ser debido a que ésta desarrolla placas de incubación y, por tanto, es más eficiente en la trasmisión del calor tanto a los huevos como a los pollos, cosa que no pasa con el macho. Mientras tanto, el macho se dedica a cazar y proveer de alimento a la hembra y los polluelos.

El que el macho cuente con menor peso y tamaño es defendido por algunos autores como una adaptación a mayor maniobrabilidad de cara a la caza (Bernis, 1974; Arroyo, 2000). Al tener que llevar a cabo esta tarea casi en exclusividad durante el periodo de incubación y parte de la cría, requiere mayor eficacia para estas labores (Bernís, 1974). Lo normal es que la hembra deposite dos huevos sobre el nido, aunque a veces sólo deposita uno y, en ocasiones más raras, hasta tres. La puesta media es de 1,9 huevos (Arroyo, 1990). Entre la puesta del primer y el segundo huevo, según nuestras observaciones, pueden transcurrir entre 3 y 5 días, de manera que el nacimiento del pollo mayor es anterior en 3 ó 5 días a su hermano menor. Esto va a ser determinante para la existencia de lo que se ha venido a denominar como cainismo (Díaz et al., 1993). De esta forma, el pollo más desarrollado siempre contará con más posibilidades de recibir comida y seguirá un desarrollo superior a su hermano pequeño. En el caso de que exista un escaso aporte de presas, de esta forma, la especie se asegura la supervivencia de, al menos, uno de los pollos. De hecho, según nuestras observaciones, lo normal es que uno de los dos pollos no llegue a desarrollarse. Brown \& Amadon (1968) vienen a ratificar esta realidad y en sus estudios aportan que en el $80 \%$ de las ocasiones el segundo pollo no prospera. Es decir, lo más habitual es la muerte por inanición, ataque del hermano mayor o ambas cosas a la vez. Autores como Del Moral (2009) cifra en 0,83 los pollos desarrollados con éxito por pareja. En Álava, en 2016 el 68,7\% de las parejas se reprodujeron con éxito y el número medio de pollos viables por pareja y año ascendió a 0,91 , igual a lo que nosotros mismos hemos visto estos dos años dentro del área de estudio. Por su parte, esto supone 0,62 pollos por pareja en relación con toda la población de Alava (Illana et al., 2016).

Al eclosionar los huevos, los pollos nacen con un plumón blanco que se mantendrá durante las siguientes tres semanas cuando comienzan a aflorar las verdaderas plumas. Los primeros 15 días son críticos puesto que los pollos no son capaces de termorregular y, en estas fechas de inicio de la primavera es esencial el aporte de calor del progenitor. Además, los adultos no se separan demasiado del nido para defender a las crías, no sólo de los rigores térmicos, sino de otros ataques o posibles riesgos. En estos primeros días la hembra se hace cargo de las presas aportadas por el macho y trocea las mismas en cachos pequeños con los que alimenta a la prole. Para la quinta semana los pollos ya estarán bastante desarrollados y serán capaces de trocear la presa y alimentarse por su propia cuenta, siempre con el aporte de presas gradual y creciente de los padres. El nido será abandonado por los aguiluchos a los 70-80 días de haber nacido aunque seguirán dependiendo de los padres. En esos momentos abandonan la estructura del nido pero se mantienen en los alrededores reclamando la provisión de alimentos y realizando vuelos cortos, que se irán ampliando en la medida en que fortalezcan los músculos relacionados con el vuelo (Pedrini \& Sergio, 2002). Durante dos o tres meses seguirán dependiendo de los padres, cada vez en menor medida, y ya estarán dispuestos para abandonar el territorio. El abandono suele ser gradual, según nuestras observaciones, de manera que pueden realizar vuelos en los que se ausentan horas o incluso días pero vuelven al territorio de los progenitores. Hasta finalizar el invierno no llevarán a cabo sus abandonos y vuelos de emancipación.

El interés del estudio de la especie en este ámbito radica principalmente en ser finícola para este distrito (Arroyo, 2003). No existen poblaciones ni contingentes aislados más al norte, dentro del sector central de la península ibérica. Se trata de una de las especies más amenazadas de la CAPV que, además, ha sido estudiada, dentro de la provincia de Álava, de forma ininterrumpida desde 1991 pero que, en los dos últimos años, no se conocen nuevos datos sobre sus contingentes poblacionales y la capacidad que tienen éstos de mantener parejas reproductoras con éxito. Ello nos ha impulsado a llevar a cabo este estudio que a través de este trabajo se expone.

El objetivo fundamental del presente trabajo es estimar y evaluar, lo más pormenorizadamente posible, los contingentes poblacionales de la especie dentro del sector occidental de Álava. Estos nuevos datos serán también comparados, en un análisis diacrónico, con los aportados anteriormente por la empresa Gadem (Illana, 1993 y 1994; Illana \& Paniagua, 1998; Illana et al., 2011 y 2016). Este fin principal puede dividirse en objetivos específicos como:

a. Conocer de primera mano la distribución de la especie dentro de este ámbito territorial.

b. Estimar sus contingentes poblacionales, a la vez que determinar cuáles pudieran ser las zonas y sectores donde nuevos contingentes pudieran afincase y prosperar potencialmente.

c. Determinar las densidades y evolución diacrónica de la especie en este ámbito a través de comparaciones con datos actuales y anteriores. 


\section{2. Área de estudio}

Para este estudio se ha tomado en cuenta un territorio que comprende 19 municipios del oeste de Álava. El área de estudio discurre desde el norte donde limita con Las Encartaciones y la también comarca vizcaína de ArratiaNervión, por el oeste con Las Merindades burgalesas, por el sur con el río Ebro y la provincia de Logroño y por el este con Los Montes alaveses, el Condado de Treviño, la Llanada alavesa y Zigiotia (Figura 2). El territorio de estudio cuenta con una superficie de $1.242 \mathrm{~km}^{2}$, con $72,3 \mathrm{~km}$ de norte a sur y $41,2 \mathrm{~km}$ de este a oeste. La altitud mínima se registra en Llodio con 110 m y la máxima con 1.481 en el Monte Gorbea, justo en el límite entre las provincias de Álava y Vizcaya (Figura 2).

En cuanto al relieve, la zona de estudio muestra una llanada o zona de topografía más amable al norte, dentro del territorio de Llodio y Amurrio, conformando la denominada Llanada de Ayala. La altitud oscila entre los 110 y los $300 \mathrm{~m}$. Este territorio discurre hasta encontrarse, de forma abrupta, con las estribaciones de la Sierra Salvada y Gorobel, con cimas dispuestas por encima de los $1.000 \mathrm{~m}$ (Urieta $1.133 \mathrm{~m}$ y Eskutxi $1.180 \mathrm{~m}$ ). Hacia el este el territorio se introduce en los hayedos de las estribaciones de Sierra Gorbea. Allí se alcanzan las máximas cotas (1.482 m en la Cruz del Gorbea). Hacia el sur aparecen las sierras de Arrato y Badaya que, a través de las Peñas de Subijana-Morillas comunican estos sistemas con la sierra de Arkamo-Gibijo hacia el oeste y, desde éstas se volvería siguiendo al noroeste hacia Gorobel. Todas estas estribaciones serranas relativamente modestas toman la forma de arco con la convexidad mirando hacia el este donde se dispone la Llanada de Badaya, sus altitudes máximas llegan a situarse ligeramente por encima de los $1.000 \mathrm{~m}$. Más hacia el oeste y antes de internarnos en la Sierra de Valderejo se dispone el valle de Cuartango con unas altitudes medias de entre 550 y $650 \mathrm{~m}$. La sierra de Valderejo discurre hacia el sur encontrándose con la sierra de Arcena. Entre las dos terminan por configurar un sistema serrano perfectamente conectado y con grandes posibilidades para el águila real. Sus cimas cuentan con altitudes máximas por encima de los $1.200 \mathrm{~m}$ (Vallegrul $1.224 \mathrm{~m}$, Revillallanos $1.297 \mathrm{~m}$, Mota $1.319 \mathrm{~m}$ o Cueto $1.362 \mathrm{~m}$ ). Discurriendo hacia el sureste nos topamos con la sierra de Añana que muestra altitudes bastante más modestas, del orden de los 700-900 m. Más hacia el sur el territorio se interna en el valle del Ebro y, por tanto, muestra un relieve no

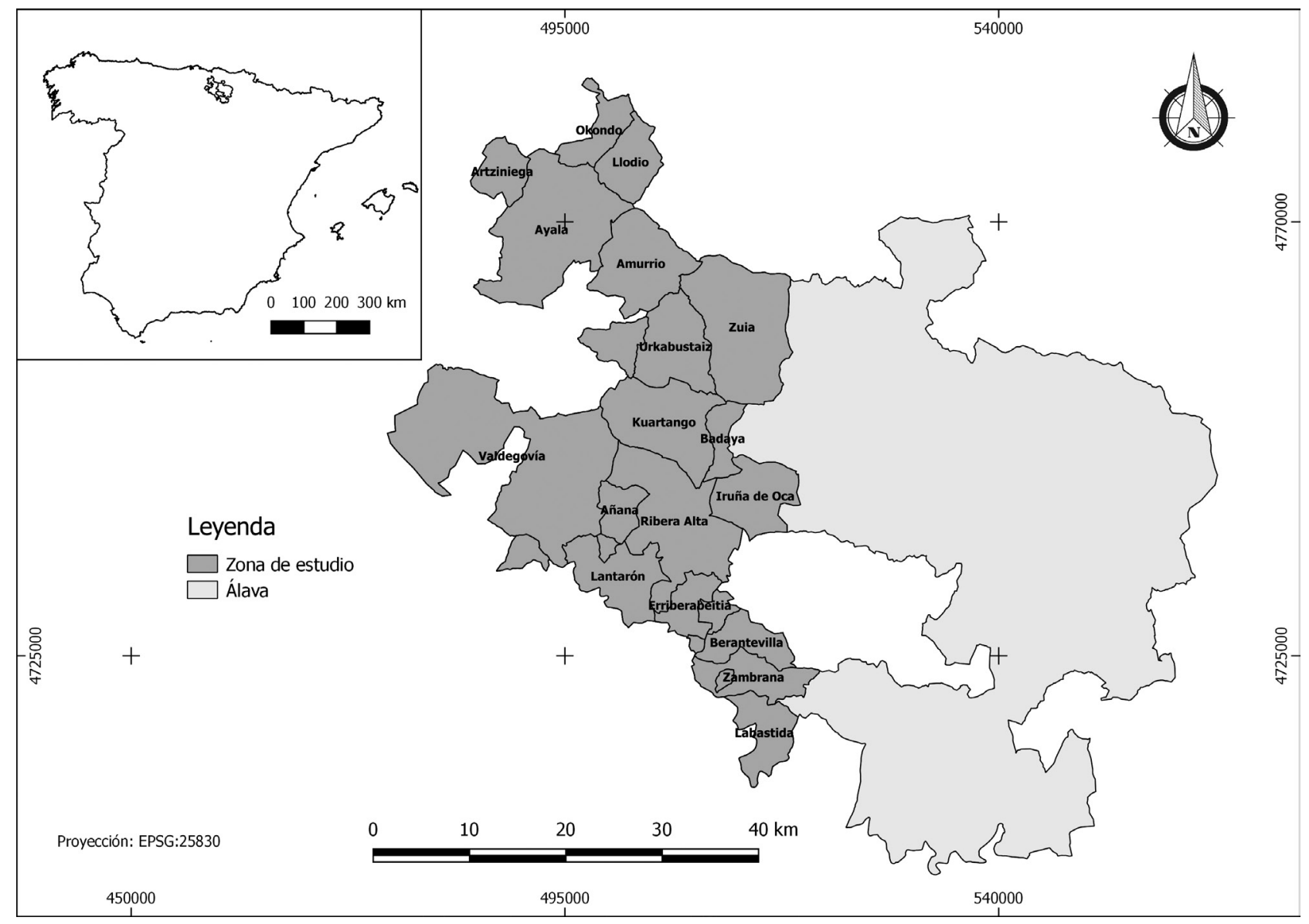

Figura 2: Localización del territorio de estudio. Fuente: Elaboración propia a partir de CNIG y Geoeuskadi. Figure 2: Location of the study area. Source: Own elaboration from CNIG and Geoeuskadi. 
tan montañoso y mucho más plano o con pendientes muy moderadas. Las altitudes medias son de 450 metros y en este sector se encuentran las partes finales o desembocaduras de ríos como el Zadorra, Bayas y Omecillo, además de pueblos como Espejo, Caicedo-Yuso y Armiñón. Más hacia el sureste volveríamos a toparnos con otro sistema montañosos relativamente modesto pero con estratos muy verticalizados $\mathrm{y}$, por tanto, muy querencioso para esta especie. Se trata de los Montes Obarenes con altitudes que no sobrepasan los $1.000 \mathrm{~m}$. Su transición hacia el este vendría dada por las estribaciones del Toloño, dentro de la denominada Sierra de Cantabria que, por espacio de $30 \mathrm{~km}$, se extiende de oeste a este aunque gran parte de este territorio queda fuera ya de la zona de estudio (Figura 3).

Las características climáticas del área de estudio son relativamente heterogéneas, puesto que hacia el norte la influencia oceánica es clara mientras que en el sector más meridional, en el valle del Ebro, las condiciones son más secas y extremas, tanto en verano como en invierno. Esta gradación se debe a la existencia de un gradiente territorial que discurre, hacia el norte, por el área de influencia del clima oceánico húmedo típico de la vertiente cantábrica. En Amurrio, por ejemplo, la temperatura media anual es de $13,3^{\circ} \mathrm{C}$, siendo enero el mes más frío, con una temperatura media de $7,5^{\circ} \mathrm{C}$ y el mes más cálido agosto con $19,7^{\circ} \mathrm{C}$. Este sector más llu-

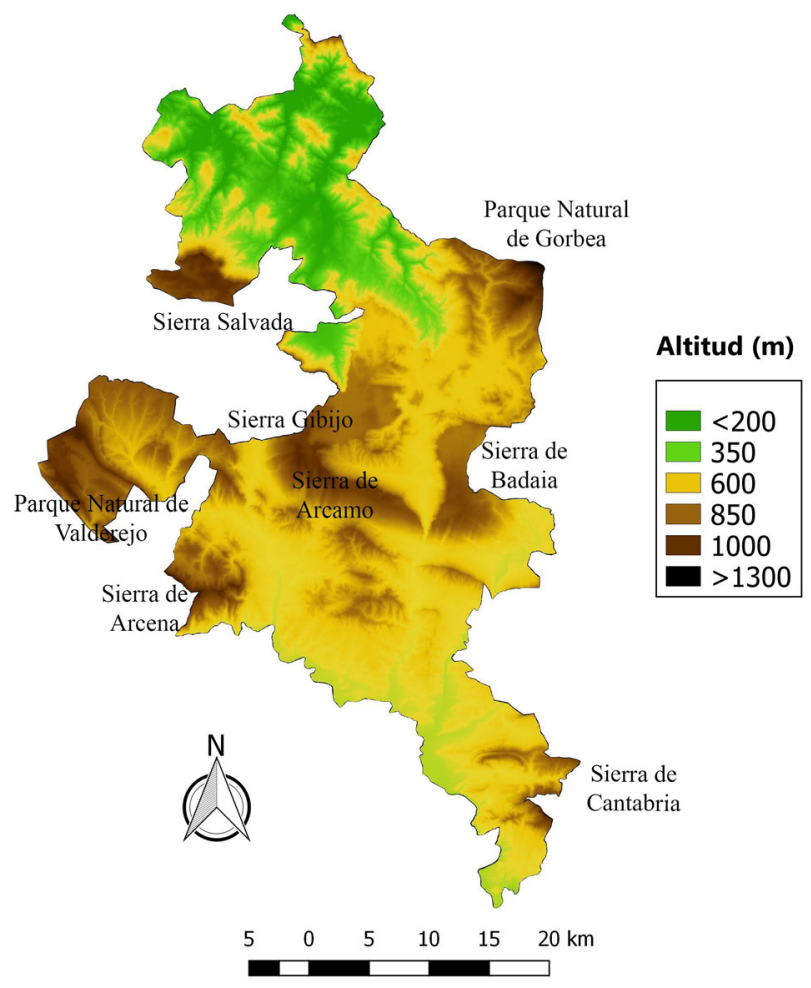

Figura 3: Mapa hipsométrico del territorio de estudio. Fuente: Elaboración propia a partir del visor de Geoeuskadi.

Figure 3: Hypsometric map of the study area. Source: Own elaboration from the Geoeuskadi viewfinder. vioso y templado aparece limitado por el umbral formado por las sierras de Gorbea, Badaya y Gibijo. A espaldas de este territorio montañoso, hacia el sur, aparece un clima con características mixtas, entre el oceánico al norte y el mediterráneo de interior al sur. En la Llanada de Badaia, en contacto con la Llanada alavesa cuya capital es Vitoria-Gasteiz, se dan temperaturas muy similares a la temperatura de esta ciudad. En este caso la temperatura media anual es de $11,2^{\circ} \mathrm{C}$, siendo el mes más frío enero con $4,4^{\circ} \mathrm{C}$ y el más caluroso julio con $18,6^{\circ} \mathrm{C}$. Por su parte, al sur, en pleno valle del Ebro podemos encontrarnos con temperaturas medias como las de Labastida (en el extremo más meridional), con $12,2^{\circ} \mathrm{C}$, siendo el mes más frío enero con $5^{\circ} \mathrm{C}$ y el más caluroso agosto con $20,1^{\circ} \mathrm{C}$, una décima más que la registrada en julio (Uriarte, 1996; Lozano \& Latasa, 2016). Como se puede observar, las temperaturas medias no muestran, en general, una gran diferencia, sin embargo, la amplitud térmica y las temperaturas son más extremas cuanto más al sur discurrimos, aunque en la zona de transición las temperaturas mínimas extremas son un poco más frías, todavía, que en la zona más meridional. El gran contraste se da con respecto a las precipitaciones. Éstas se reparten de forma más homogénea al norte, mientras al sur se hacen más escasas y con un régimen más torrencial, típico del clima mediterráneo de interior. Mientras en Amurrio las precipitaciones medias alcanzan los $1.000 \mathrm{~mm}$, en Vitoria-Gasteiz llegan a $828 \mathrm{~mm}$ y en Labastida a $662 \mathrm{~mm}$. En este caso, se ve claramente la transición desde un clima típicamente oceánico, con precipitaciones bien repartidas a lo largo de todos los meses (sin que exista una verdadera sequía estival), pasando por un clima de transición, hasta alcanzar los mínimos precipitacionales en el sector más meridional del territorio, con un régimen claramente seco, una estación cálida y seca como es el verano y unos máximos precipitacionales que, aunque escasos, se distribuyen desde finales del otoño hasta la primavera, teniendo su máximo en invierno. De esta forma, mientras al norte existiría un clima $\mathrm{Cfb}$ (según la clasificación de Köppen), en el sector más meridional se registraría un clima Csa o mediterráneo y, en el sector central otro de tipo $\mathrm{Cfb} / \mathrm{Csb}$ que podríamos definir como subatlántico o submediterráneo, según nos situemos más al norte o al sur (Uriarte, 1996). En cualquier caso, una buena parte del territorio cuenta con un claro carácter climático transicional. A ello hay que sumar la existencia de todo ese rosario de sierras que confieren otras características climáticas a aquellos ámbitos donde se sitúan. En general, en estos ámbitos serranos las temperaturas son más frescas y las precipitaciones más altas de las que les corresponderían en su distribución zonal (Lozano \& Latasa, 2016).

En cuanto a los usos del suelo (Figura 4), lo cierto es que se llegan a registrar hasta 11 formaciones o paisajes diferentes. En las zonas más planas y, teniendo en cuenta que de norte a sur existe la descrita transición climática entre un régimen templado oceánico a otro totalmente mediterráneo, se disponen cultivos y pastos. Al norte do- 


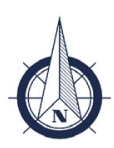

Leyenda

Bosques

Rios y embalses

Suelo desnudo

Cultivos agricolas

Prados y pastizales

Matorral

Parques urbanos

Plantaciones forestales

Urbano

Proyección: EPSG: 25830

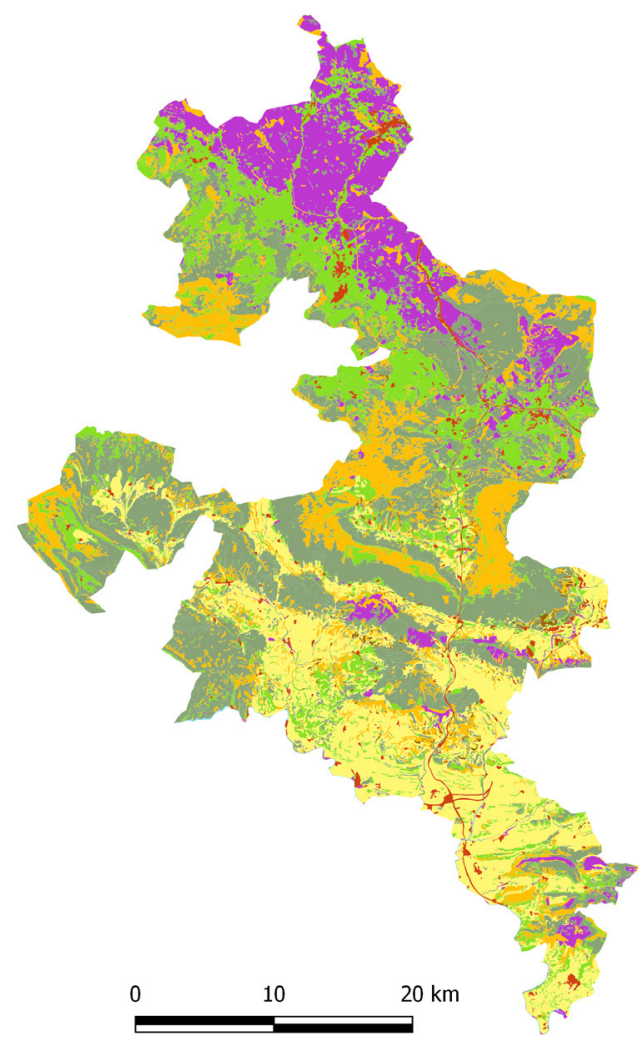

Figura 4: Grandes Usos del Suelo del territorio de estudio. Fuente: Elaboración propia a partir de Coryne Land Cover (2018) y Geoeuskadi.

Figure 4: Main Land Uses of the study area. Source: Own elaboration from Coryne Land Cover (2028) and Geoeuskadi.

minan más los pastos ganaderos aunque también se encuentran zonas de huerta y cultivos como el maíz, la remolacha e incluso la patata. Al sur, lógicamente, dominan los campos de cereal y la vid. En la parte central, en Llanadas como la de Badaya o el valle de Cuartango domina el cereal pero también cultivos como la patata y la remolacha. En las sierras del noreste, fundamentalmente las estribaciones del Gorbea, dominan las plantaciones de exóticas como Pinus radiata. Éstas se alternan con importantes extensiones de hayedo ya a cotas por encima de los 700-800 metros. Al noroeste, por su parte, discurriendo desde el valle de Ayala a las estribaciones serranas de Sierra Salvada existe una transición desde los quejigares de Quercus faginea hacia los brezales montanos. En el sector central, precisamente ocupando la zona de transición climática entre el mundo oceánico y el mediterráneo de interior, se disponen importantes extensiones de quejigo (Quercus faginea) con bosques de roble carvallo (Quercus robur) en los suelos más profundos, frescos y húmedos de La Llanada Alavesa. La transición de estas formaciones hacia el encinar (Quercus ilex), es un hecho en las zonas más bajas y meridionales, al igual que la presencia de los mencionados campos de cultivo dominados por el cereal. Al oeste, sin embargo, en ámbitos serranos como los de Valderejo, Arcena pero también en los modestos montes de Añana existen importantes extensiones de pinares naturales de Pinus Sylvestris. En el valle del
Ebro y las desembocaduras del Bayas, Zadorra y Olmecillo se imponen los campos de cultivo y las vides y en los cursos fluviales la vegetación de ribera. Ya en los Obarenes y Toloño volvemos a toparnos con plantaciones forestales de exóticas, encinares (Quercus rotundifolia) y quejigares en las partes bajas y hayedos en las altas. En algún pequeño sector de la Sierra de Elgea, en el sector más oriental dentro de la zona central, se pueden encontrar algunas extensiones de rebollo (Quercus pyrenaica) (Meaza et al., 2020).

Para este taxón (Aquila chrysaetos) la presencia humana supone un freno o una cortapisa, de manera que también se ha estudiado y se describirá de forma sucinta la densidad de población y su reparto territorial. En general, en toda la zona de estudio las densidades de población humana son bajas. Los municipios con mayores poblaciones se sitúan al norte (Llodio, 18.249 hab y Amurrio, 10.336 hab) (Eustat, 2016), a excepción del municipio de Iruña de Oca que se sitúa al este. En el resto del territorio las densidades son de bajas a muy bajas oscilando entre los 4 y $5,5 \mathrm{hab} / \mathrm{km}^{2}$. Son precisamente en esos entornos ubicados en los sectores centrales y meridionales donde se dan los mayores contingentes poblacionales del águila real. Existe una correlación directa y positiva entre las zonas con menores densidades poblacionales del ser humano y las mayores densidades del taxón. Por otra parte, otro de los elementos negativos para el taxón serían las grandes infraestructuras. 
La red más densa de carreteras también se circunscribe a la parte norte, el valle de Ayala, municipios como Llodio y Amurrio, de tal manera que a las densidades de población humana más altas de toda el área de estudio se le suman el mayor número de kilómetros de carreteras de alta y media capacidad. En cualquier caso, la infraestructura más importante es la Ap-68 que discurre entre Zambrana al sur, y Amurrio al norte, lo hace por las zonas más bajas y no parece suponer mayor obstáculo para el águila real que, en esta zona en general, centra sus territorios precisamente fuera de esta gran infraestructura.

\section{Metodología}

A través de un complejo pero muy contrastado proceso metodológico derivado de transectos regulares realizados durante dos años (entre enero de 2017 y enero de 2019 -850 horas de trabajo de campo aproximadamente-) y acompañados de visitas a lugares sensibles junto a estaciones de vigilancia, se ha realizado la estimación poblacional a la vez que se han estudiado otras variables como la dieta (basada en las observaciones obtenidas a la vista de los aportes realizados a los nidos y los restos situados debajo de las perchas -siempre de forma cualitativa-), la ubicación de los nidos y las parejas, los movimientos de dispersión y las posibilidades de distribución de la especie en el futuro dentro del ámbito territorial mencionado.

El águila real muestra dificultades evidentes a la hora de su estudio. En primer lugar cuenta con territorios extensos y relativamente accidentados. Muchas veces se encuentran encaramados a un risco de manera que es difícil su localización exacta. A ello hay que añadir que, aunque formen parejas estables, la mayor parte del tiempo cada uno de los miembros de la pareja desarrolla sus propias actividades de manera que, salvo en el cortejo, es difícil diferenciar la existencia o no de la pareja. Sólo con observaciones sistemáticas, largas y continuadas se puede llegar a la determinación de parejas estables sobre un territorio. Para establecer dicha cuestión, precisamente, se realizó un esfuerzo adicional en época de celo, entre enero y marzo de 2018. Por otra parte, los jóvenes en dispersión y, a veces, incluso adultos sin pareja o recién llegados a la época reproductora, realizan largos y extensos desplazamientos que pueden llegar a los cientos de kilómetros. Para evitar estos sesgos y no falsear las parejas y territorios ocupados se tomaron las siguientes determinaciones: sólo se constataba una pareja sobre un territorio dado cuando se divisaban dos ejemplares de diferente sexo volando a la vez y de forma relativamente emparejada; cuando se divisaba un adulto incubando los huevos sobre el nido o cuando se constataba la existencia de un adulto alimentando al pollo. Sólo en estos casos se daba por buena la existencia de una pareja reproductora sobre un territorio concreto.

En segundo lugar se ha tenido en cuenta el trabajo de Illana et al. (2016). En este informe se determinaban las parejas y los contingentes existentes para todo Álava. Basados en este trabajo, siempre que se ha divisado un ejemplar en un territorio determinado con anterioridad pero no se han constatado otro tipo de pruebas más contundentes, se ha tomado en cuenta el mismo como "supuesto territorio con pareja".

Tal y como se referenció anteriormente, las observaciones directas se realizaron a través de dos técnicas básicas como son las estaciones de observación y los transectos (Illana et al., 2016). Los primeros se establecieron en aquellos puntos relevantes del relieve por su alta visibilidad. Estos puntos de observación fueron 21 y se determinaron, no sólo por su alta visibilidad, sino que se intentó cubrir la mayor parte del territorio y repartirlos de forma aleatoria pero estratégica. Para aquellos sectores más llanos o con cubierta forestal donde la visibilidad era inferior se determinaron transectos. Éstos también han sido 21 (Figura 5). En cualquier caso, tanto para los transectos como para las estaciones de observación se han registrado visitas prácticamente cada 21 días y en cada una de ellas se establecían estancias o recorridos de entre 30 y 45 minutos. Para facilitar la observación y la determinación de las parejas se han utilizado tanto un telescopio terrestre (Kowa TSN-821M) como unos prismáticos (Zeiss 10x40 T* FL).

Para determinar cuáles son las principales fuentes alimenticias de la especie también se han puesto en marcha

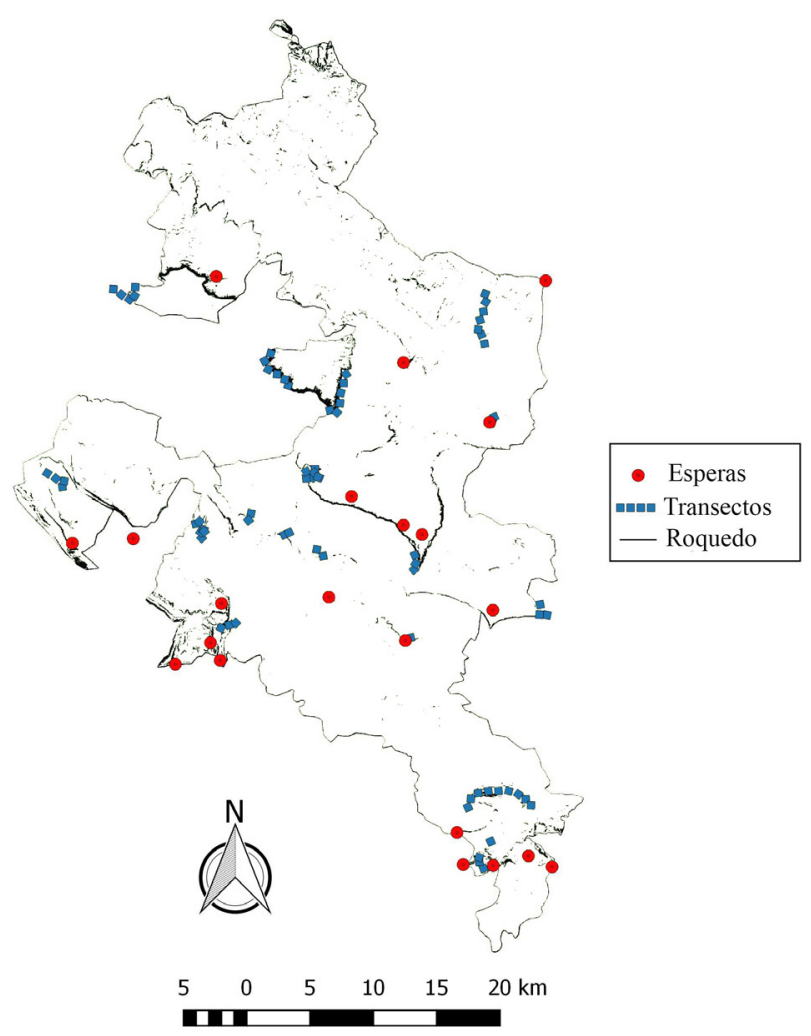

Figura 5: Localización de las estaciones de observación, los transectos y las paredes rocosas o cortados más importantes: Elaboración propia a partir del visor de Geoeuskadi.

Figure 5: Location of the most important observation stations, transects and main rocky or cut walls. Source Own elaboration from the Geoeuskadi viewfinder. 
una serie de herramientas metodológicas relativamente sencillas pero que requieren de una gran cantidad de visitas a lugares sensibles y, con ello, muchas horas de acceso y observación. En primer lugar y, a partir de las observaciones abundantes y prolongadas en tiempo, se determinaron los posaderos y perchas más utilizadas por cada una de las parejas estudiadas (de media, para cada pareja se estimaron 6 perchas diferentes). Se realizaron visitas regulares a dichas perchas (cada 15 días en época de cría) $\mathrm{y}$, en esas visitas, se registraban todos aquellos rastros que pudieran darnos pistas sobre las presas predadas: plumas, pelos, huesos, partes de cadáveres, etc. En este caso, se recogían todo ese tipo de rastros para no contar con un sesgo en posteriores visitas. De esta forma, cada posadero se dejaba libre de cualquier rastro detectado en cada una de las visitas. Además de eso, se estimó como oportuna la realización de visitas extremadamente discretas a atalayas de observación cercanas a los nidos en época de cría (Díaz et al., 1983). En este caso, se pasaba un total de entre 4 ó 5 horas de observación en cada uno de los 6 nidos y dos o tres veces durante la mencionada época de cría y ceba y para cada uno de los nidos. En cada una de esas estancias se apuntaban los aportes al nido de los adultos, fueran el macho o la hembra, normalmente, hasta en un $76 \%$ de las ocasiones fue el macho. A través del telescopio se pudo identificar perfectamente cada uno de los aportes realizados en cada uno de los 6 nidos identificados y estudiados.

\section{Resultados y discusión}

Los resultados obtenidos a través de la batería metodológica se han centrado en dos aspectos fundamentales. Por una parte determinar el número de parejas reproductoras existentes en la zona y territorios limítrofes y en segundo lugar cómo se reparten.

\subsection{Número de parejas reproductoras y territorios ocupados}

Los resultados hablan de una distribución poblacional muy en consonancia con los requerimientos de la especie. Sólo aparece en aquellos sectores con gran cantidad de presas, poco impactados por el ser humano, con poca presión antrópica y con un paisaje en el que alternan bosques bien conservados junto a espacios abiertos de campiña y pastos (Illana et al., 2011 y 2016). Seis han sido los territorios y parejas confirmadas mientras que dos no han podido ser confirmados a partir del presente estudio. Los territorios constatados dentro del área de estudio son Arriano, Karanka, Barron, Hereña y Gatzaga-Buradon, el sexto, por su parte se sitúa fuera de la zona de estudio dentro de Angulo, en el nordeste de la provincia de Burgos. Sin embargo, la pareja ocupante de este territorio hace abundantes incursiones dentro del territorio alavés sobre el que tiene gran parte de su zona de campeo, de manera que se ha adoptado como pareja residente o, como mínimo, usufructuaria del área y sus recursos.

Teniendo en cuenta el mencionado estudio de Illana et al. (2016) también se han podido constatar otros dos territorios definidos como "supuestos" puesto que no se ha podido detectar a la pareja, sino que se han avistado individuos solitarios en ambos casos. Se trata de SubijanaMorillas y Aprikano. En este último caso, efectivamente, sólo se pudo constar la presencia de un individuo aunque hay que tener en cuenta que está catalogado como nido histórico desde 1990 (Illana, 1991). En el primer caso también se trataría de un nido histórico fuera de territorio Alavés, dentro de la provincia de Burgos pero que, también fue constatado por el trabajo de Illana et al. (2016). Aunque el nido se encuentre fuera del área de estudio buena parte del territorio de campeo y caza de la pareja se dispone dentro (Figura 6).

En resumen, se puede afirmar que la cantidad de parejas reproductoras que se sitúan en el área de estudio puede oscilar entre 6 y 8 , sin tener en cuenta otros avistamientos de ejemplares en dispersión o no emparejados. $\mathrm{Al}$ respecto, hay que reseñar que, aparte de las parejas reproductoras, también han sido detectados otros dos individuos solitarios en territorios no ocupados hasta la fecha por ninguna pareja. Uno en las inmediaciones del Santuario de Oro, en el término municipal de Zuia pero que se ha ido moviendo por los alrededores, siempre en las in-

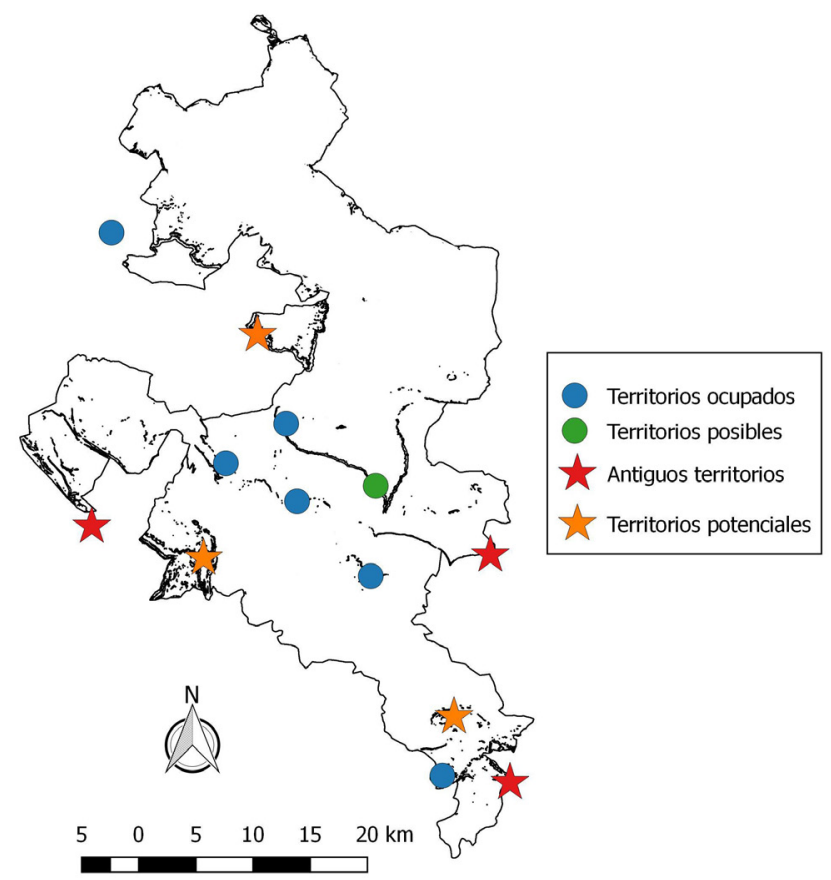

Figura 6: Localización de los territorios ocupados y posibles en la actualidad, así como aquellos territorios históricos y los potenciales. Fuente: Elaboración propia a partir del visor de Geoeuskadi.

Figure 6: Location of the occupied and possible territories today as well as those historical territories and potential ones.

Source: Own elaboration from the Geoeuskadi viewfinder. 
mediaciones del Parque Natural del Gorbea, y otro en Ocio, en las cercanías de la Sierra Portilla. En el primer caso, se trataba de un ejemplar joven, seguramente en dispersión. Aunque el Santuario de Oro y sus alrededores cuentan con ambientes rocosos y otras especies de aves rupícolas aparecen en él, no parece un lugar adecuado para que una pareja pueda asentarse y prosperar, entre otros aspectos porque la presencia y presión antrópica es superior a la detectada en aquellos territorios ocupados por la especie a día de hoy y, además, las poblaciones de las presas potenciales tampoco son muy elevadas. Al respecto, dentro de estos ambientes más atlánticos e incluso montanos las poblaciones del conejo (Oryctalagus cuniculus), la perdiz (Alectoris rufa) e incluso la paloma torcaz (Columba palumbus) se reducen al máximo mientras que la liebre ibérica (Lepus granatensis) desaparece (Illana et al., 2016). Es cierto que, en su defecto, existen magras poblaciones de Lepus europaeus (Palomo \& Gisbert, 2002) pero ésta, sin embargo, muestra un carácter más forestal que la primera y en ese ambiente es difícil que el águila real pueda entrar a realizar sus capturas. En el segundo caso, era un ejemplar adulto y este entorno sí reúne las condiciones adecuadas para el establecimiento de una pareja y la creación de un nuevo territorio. Sin embargo, sólo fue avistado en enero de 2018 y con posterioridad desapareció.

En números absolutos estas cifras serían bajas comparadas con las 95 parejas de la provincia de Jaén o las 94 de Zaragoza. No obstante, en números relativos, teniendo en cuenta los $\mathrm{km}^{2}$ y las parejas asentadas en ellos, las densidades poblacionales son realmente elevadas, del orden de 0,48 parejas $/ 100 \mathrm{~km}^{2}, 0,62$ parejas $/ 100 \mathrm{~km}^{2}$ si tenemos en cuenta también las parejas que no han podido ser confirmadas. Con estas cifras estaríamos en disposición de afirmar que supondrían una de las densidades mayores de toda España. Para realizar una comparación habría que tener en cuenta que las densidades más altas se dan en la provincia de Jaén con 0,68 parejas $/ 100 \mathrm{~km}^{2}$, La Rioja y Teruel las dos con 0,64 parejas $/ 100 \mathrm{~km}^{2}$ y Guadalajara con 0,58 parejas/100 $\mathrm{km}^{2}$ (Del Moral, 2009). Si tomamos en cuenta solamente los territorios certificados por nuestro trabajo, la zona de estudio se situaría en el $13^{\circ}$ lugar en cuanto a densidad de población de la especie dentro de España. Sin embargo, si tomamos en cuenta también los "supuestos" dos territorios más, la posición sería la 4".

En el caso de la ubicación de los nidos, éstos se ubicaron en cortados relativamente grandes (más de 50 metros) aunque uno se ha ubicado en un cortado relativamente modesto (menos de 20 metros de pared). La envergadura de los nidos es, en general, relativamente modesta, aunque en alguno de ellos alcanzó casi dos metros. Además, los materiales más recurridos han sido, en cuanto a aquellos más blandos, pequeñas ramitas de pino rojo (Pinus sylvestris), junto a otros aportes de coníferas y, en menor medida, de especies como la encina (Quercus rotundifolia) y el quejigo (Quercus faginea). Aunque el aporte de estos y otros materiales más gruesos se intensifica durante la época de cortejo y cría (febrero y marzo especialmente), lo cierto es que los individuos de las diferentes pare- jas han aportado, durante estos dos años de investigación, materiales a lo largo del año.

Hay algunos autores que afirman que las parejas no suelen utilizar los mismos nidos para evitar posibles contagios de enfermedades a los pollos al quedar llenos de residuos de presas después de la cría (Brown y Amadon, 1968; Arroyo, 2000). Sin embargo, en nuestro caso, hemos observado que lo más habitual es que las parejas vuelvan al mismo nido si la cría del año anterior resultó exitosa. El caso del nido de Hereña es un buen ejemplo. Durante los años 2017 y 2018 se constató la cría con éxito de la pareja allí ubicada. Los dos años sacaron adelante un único pollo dentro de un nido de gran volumen y con un aporte de materiales y una envergadura que parece indicar que la costumbre de esa pareja a criar dentro de ese nido no viene, simplemente, de esos dos años, sino que es más prolongada en el tiempo (Figura 7). No obstante, de media, para Álava cada pareja viene a utilizar cada periodo de cría 3,9 nidos activos diferentes (Illana et al., 2016). Es decir, la pareja se dedica a construir casi 4 nidos o a depositar materiales diversos en casi 4 ubicaciones diferentes. Al final escogerá una y esa será donde lleve a cabo la puesta, la incubación y la cría de los pollos.

\subsection{Distribución espacial de las parejas y los nidos}

En lo referente al reparto territorial de las parejas, aquí existe una clara segregación puesto que hasta un $75 \%$ de la población se sitúa en el sector central del territorio, en esa banda bioclimática que podemos definir como de transición o submediterránea. Junto al resto de territorios que se situarían claramente dentro del área mediterránea, éstos son los que mejores condiciones ecológicas, fundamentalmente tróficas, registran, a tenor de nuestra investigación. En estos sectores se darían las densidades poblacionales más altas de presas clave para la especie como son la liebre ibérica (Lepus granatensis), el conejo (Oryctolagus cuniculus) o la perdiz roja (Aleactoris rufa). Estas tres especies conforman más del $62 \%$ de la dieta de las parejas estudiadas (Illana et al., 2016).

Otro requerimiento importante para explicar la segregación o el reparto territorial es la presencia de sierras y sistemas montañosos. No obstante, el águila real no sólo ocupa las sierras más elevadas o masivas, sino que en el área de estudio se la puede localizar en sierras más bajas y modestas como es el caso de Cantoblanco y Peña Grajera (1.006 y $832 \mathrm{~m}$., respectivamente). La condición que indefectiblemente debe cumplirse es la de contar con cortados y paredes rocosas de cierta entidad. Aunque prefieren taludes amplios y altos, lo cierto es que también pueden ocupar algunos más modestos, por lo menos en la zona de estudio. En el caso de Hereña, el nido fue situado en una pared rocosa pequeña y baja.

Además de estos requerimientos también es importante determinar que la especie busca, sin lugar a dudas, aquellos enclaves donde la presencia humana o los disturbios ocasionados potencialmente por ella son inexistentes o muy reducidos. Esto se hace palpable en épocas como 


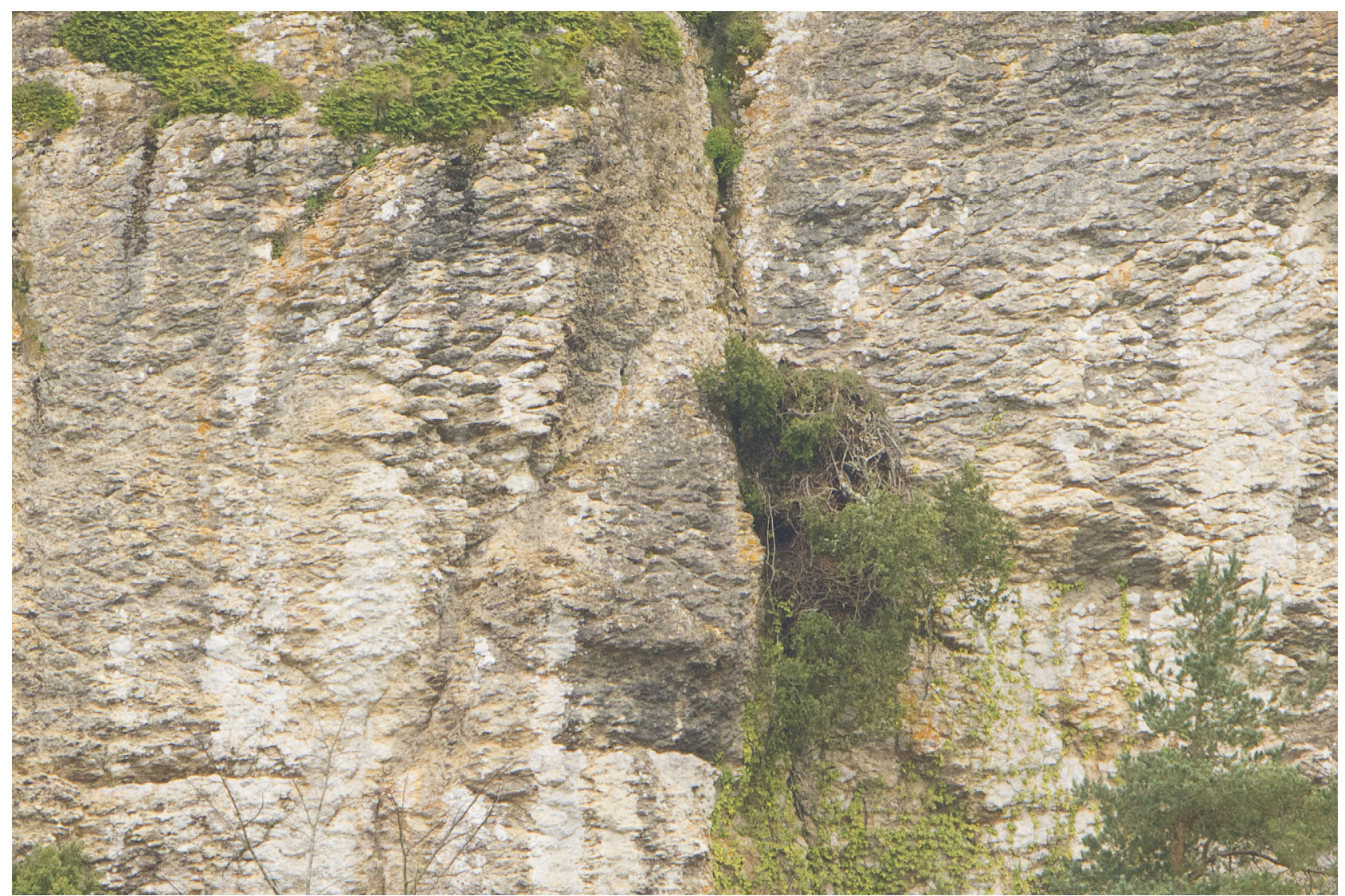

Figura 7: Estructura de nido en la que se ha venido localizando la puesta y la cría durante una serie dilatada de años. La hembra se encontraba incubando. Fotografía de los autores.

Figure 7: Nest structure in which the laying and breeding has been located for a long series of years. The female was incubating. Photograph of the authors.

el cortejo pero, sobre todo, la incubación y la cría. En la década de los 90 del pasado siglo el 9,3\% del abandono o fracaso de la pollada fue por motivos antrópicos (Illana et al., 2016). Sin embargo, Illana \& Paniagua (1998) afirman que detrás de esos fracasos, hasta en un $43,75 \%$ de las ocasiones se sitúan destrozos intencionados del nido, robo de huevos y/o pollos, excesivo acercamiento de los investigadores o fotógrafos a los nidos, molestias generadas por el paso o actividades de montañeros y escaladores o aquellas generadas por actividades forestales. Todas estas actividades son, si cabe, más peligrosas en el momento en que los huevos eclosionan o los pollos se encuentran en sus etapas más incipientes. En este sentido, dado que se cuenta con las ubicaciones exactas de los territorios y los nidos de cría, se debería poner un especial énfasis por parte de la administración competente (Diputación Foral de Álava y Gobierno Vasco) y por el servicio de guardería para vigilar de forma exhaustiva estos emplazamientos y evitar cualquier tipo de actividad que pueda disturbar, en época de celo, reproducción y/o cría el éxito reproductor de la especie.

Por otra parte, no parece que las carreteras o los trazados ferroviarios tengan un especial impacto en las parejas asentadas. Así parece a la vista del éxito reproductor de las parejas de Aprikano y Hereña. En este último caso, el nido se sitúa a escasos 150 metros de la Ap-68 y el trazado ferroviario Madrid-Irún. En algunos casos los nidos tampoco están demasiado alejados de los núcleos urbanos, aunque éstos son pequeños y poco poblados. En este último caso, se encontrarían las localizaciones de Karanka, Barrón y Hereña. Las actividades humanas rutinarias y repetitivas no parecen contar con un alto impacto, siempre y cuando se sitúen a cierta distancia, mientras las actividades más puntuales (escalada, montañismo, limpias y cortas forestales...) sí presentan un impacto fuerte y evidente.

Otra característica que ha cumplido la ubicación de los nidos, además de situarse en paredes rocosas más o menos extensas, ha sido la de situarse bajo bosques cuya cobertura forestal es notable, es decir, bosques relativamente tupidos y maduros. Todos los nidos se han situado en sectores boscosos aunque en las inmediaciones existen importantes extensiones de cultivos y zonas despejadas. En esta especie se da una clara segregación territorial o de usos del suelo, puesto que tiende a ubicar sus nidos y posaderos o perchas favoritas en zonas boscosas, mientras que sus territorios de campeo y caza son mayoritariamente abiertos, en forma de prados pero, sobre todo, tierras de 
cultivo. Especial relevancia muestran los campos de cultivo en régimen de secano, puesto que a principios del verano son muy visitados por las tres especies clave antes citadas, y otras como la paloma bravía (Columba livia) y la paloma torcaz (Columba palumbus), absolutamente estratégicas para la alimentación del águila real.

\subsection{Potencialidad del territorio para la radicación de nuevas parejas}

En cuanto a la dinámica futura de la especie en este área, hay que decir que la población del taxón va variando continuamente a lo largo del tiempo. En algún caso, si el territorio que ha quedado vacante reúne las condiciones adecuadas, al poco tiempo será ocupado por otros individuos u otra pareja (Díaz et al., 1996; Del Moral, 2009). En otras ocasiones, uno de los dos individuos de la pareja muere o desaparece y el otro se queda esperando a otro individuo del sexo contrario. De esta forma, hay territorios que se mantienen durante años pero en los que los individuos van cambiando, de manera que existe un continuo flujo de restauración o renovación. En el caso de la provincia de Álava la tendencia ha sido progresivamente positiva. En 1990 se conocían 11 territorios con sus respectivas parejas (Illana, 1991); en 1993, 13 (Illana, 1994); en 2010, 19 (Illana et al., 2011), y en 2016, 18 (Illana et al., 2016). De esta manera, aunque en los últimos 6 años se ha perdido un territorio, la población de Álava ha crecido entre 1990 y la actualidad un 63,6\%, mostrando 7 territorios nuevos ( 8 en 2010), lo que supone un crecimiento bastante considerable. La explicación a este incremento pasa por atestiguar el aumento de las poblaciones de algunas especies clave como el conejo (Oryctolagus cuniculus) o la liebre ibérica (Lepus granatensis) pero, sobre todo, por la concienciación ciudadana y el control administrativo (Illana et al., 2016). La caza, el veneno, las trampas y la destrucción de nidos o la sustracción de huevos o pollos se han reducido drásticamente. Según los datos de la Diputación Foral de Álava con respecto a los individuos de $A$. chrysaetos ingresados dentro del centro para la recuperación de fauna salvaje de Martioda, solamente dos ejemplares provenientes de la zona de estudio han sido ingresados en los 10 últimos años. Uno de ellos provenía de Sobrón y había sido tiroteado, aunque fue curado y volvió a volar en libertad, y el segundo, de Sendadiano, ingresó cadáver al haberse electrocutado con un cable de alta tensión. En los dos casos los individuos eran adultos o reproductores, y ambos accidentes sucedieron el año 2010. Es posible que haya habido algún otro caso de muerte que, sin embargo, no ha sido identificado o no ha sido notificado, pero lo cierto es que las mortalidades por causas traumáticas o antrópicas se han visto reducidas considerablemente (Illana et al., 2016). Otro factor importante es el incremento en la protección de espacios y ecosistemas. En el área de estudio se han calificado como protegidos el Parque Natural de Gorbea, el Biotopo protegido del diapiro de Añana, la Zona de Especial Protección para las Aves de Arkamo-Gibijo, la Zona de Especial
Conservación de las Sierras Meridionales de Álava, etc. Este incremento ha beneficiado a la especie procurándole hábitats con una recuperación vegetativa importante hacia bosques de gran riqueza y también evitando actividades antrópicas impactantes o que generaban disturbios hacia el taxón. Esta mayor protección del área de estudio ha influido en su conservación y en que la población de esta zona se esté incrementando considerablemente.

A la vista de las necesidades de la especie, se puede afirmar que, aunque su crecimiento poblacional en estos últimos años haya sido notable, no existen mayores posibilidades de nuevos emplazamientos para otras parejas. Es muy probable que la especie mantenga sus contingentes poblacionales actuales o se incrementen ligeramente a la vista de citas de nidos, parejas o territorios existentes anteriormente pero que no han podido ser constatados. Al respecto, en la figura 6 aparecen reflejados aquellos territorios potenciales para el establecimiento de nuevas parejas. De esta manera, podemos observar que los territorios ocupados hoy en día son los 6 anteriormente referenciados y los potenciales nuevos territorios, muy relacionados con la existencia de cortados, buenas condiciones tróficas, cobertura mixta de bosques maduros, pastizales y tierras de cultivo y escasas interferencias antrópicas, pueden ser otros 3 , a los que se pueden sumar otros tantos derivados de antiguas localizaciones de parejas históricas. Entre Tuyo y Subijana existía una pareja entre 2010 y 2016 (Illana et al., 2016), en el Toloño también fue descrito un territorio y una pareja desde hace más de dos décadas (Illana, 1991; Illana et al., 2016) cuya pervivencia se ha podido constatar, por lo menos, hasta 2016. Por último, también se conocía un tercer territorio o pareja en las inmediaciones de Valderejo (Illana et al., 2016). En este caso, los nidos se situaban en la provincia de Burgos pero la pareja prospectaba muy a menudo el territorio alavés. En cualquier caso, a través de nuestra investigación no se ha podido constatar que hoy en día estos territorios estén ocupados y, por tanto, se pueden considerar como entornos potenciales para la instalación de nuevos individuos o parejas. En lo que respecta a los denominados como "territorios potenciales" estos abarcarían la Sierra de Arcena, muy cerca de su pico más elevado (Batxikabo, 1.199 $\mathrm{m}$ ), Sierra Salvada (entre Tologorri y Eskutzi) y, por último, al sureste del Monte Txulato, en la Sierra de Portilla.

Quizá el emplazamiento potencial más adecuado de los tres citados sería el segundo, aquel centrado en los roquedos del Monte Gorobel. Hay buena cobertura boscosa, existen buenas paredes rocosas y en las inmediaciones hay zonas abiertas y suficientes recursos tróficos así como pocos disturbios antrópicos, por lo que aunque con nuestra investigación no se han encontrado parejas, pero sí individuos aislados y citas históricas de la especie, este espacio parece adecuado para que nuevas parejas puedan asentarse en él. En el Valle de Cuartango ya existen dos parejas, una confirmada y la otra no constatada por nuestro trabajo de campo. No obstante, estimamos que por sus condiciones, sus abundantes paredes y la potencia de recursos, podría albergar, en un futuro, una tercera pareja. En la Sierra de Arcena, el primer sector potencial citado, 
también podrían emplazarse nuevas parejas debido a lo extenso del territorio y las grandes paredes rocosas que presenta. No ocurriría lo mismo con la Sierra de Gorbea. Lo cierto es que existen numerosas citas de individuos aislados, y más o menos en dispersión, pero el espacio no cuenta con roquedos de entidad, aunque sí con un gran nivel de protección y buena cobertura arbórea. En lo que respecta a los recursos tróficos, hay que reseñar que nos encontramos en el límite más septentrional de la zona bioclimática de transición y, por tanto, las poblaciones de las especies clave se van reduciendo considerablemente. No obstante, nunca ha sido citado como un espacio con pareja estable (Zuberogoitia \& Torres, 1997; Illana et al., 2011 y 2016). Por último, el tercer enclave potencial de gran interés antes reseñado podría ser la Sierra de Portilla. En primer lugar cuenta con buenos roquedos, en segundo muestra una superficie forestal muy interesante y, en tercero, se encuentra colindante con una gran finca de caza que, regularmente, "siembra" el coto con especies de caza menor que son muy indicadas para el águila real. Éstos contingentes suelen huir, en parte, de manera que el recurso trófico, en este caso, estaría perfectamente garantizado. A parte de esto, cuenta con buenas poblaciones de las especies clave para la dieta del águila real (conejo, liebre, paloma torcaz, paloma bravía, etc.).

\subsection{Otros aspectos de la dieta y ecología}

En cuanto a uno de los aspectos más notables de su ecología, la dieta, hay que afirmar que, según nuestras observaciones, esta especie es muy adaptable, de manera que puede apresar y predar sobre especies tan voluminosas como el corzo (Capreolus capreolus) (sobre todo ejemplares jóvenes) pero, a su vez, llegar a apresar taxones tan pequeños como el mosquitero común (Phylloscopus collybita) (Bautista et al., 2016). En el área de estudio se han podido determinar predaciones sobre especies ya citadas con anterioridad como recursos tróficos. Sin embargo, más interesantes son los resultados del seguimiento a uno de los nidos concretos, el de Cantoblanco. Los apresamientos atestiguados fueron los siguientes: un arrendajo (Garrulus glandarius), una paloma torcaz (Columba palumbus), una paloma bravía (Columba livia), dos liebres (Lepus granatensis) de las que una fue llevada al nido justo en el momento del rececho, un macho de azulón (Anas platyrhynchos), una chova piquirroja (Pyrrhocorax pyrrhocorax) y un córvido que no se pudo identificar con claridad, pero que seguramente era una corneja común (Corvus corone). Además de éstos se pudieron atestiguar también un intento de caza sobre un arrendajo (Garrulus glandarius) y sobre una paloma torcaz (Columba palumbus) no resultando, en los dos casos, fructíferos. Con ello y con los avistamientos generales realizados estamos en disposición de asegurar que la mayor parte de las especies sobre las que preda el taxón se corresponden con animales forestales o semiforestales (corzo, arrendajo, córvidos, paloma torcaz) pero, a su vez, también preda abundantemente sobre especies de espacios abiertos como conejos, liebres, perdices, palomas bravías, etc. Por ello es tan importante que los territorios donde se encuentren las parejas o aquellos que presentan una alta potencialidad cuenten con sectores de bosques relativamente maduros, bien conservados e intricados y otros espacios abiertos como tierras de cultivo, pastizales e incluso matorrales bajos.

\section{Conclusiones}

El águila real, en el ámbito de estudio, aparece en aquellos sectores con buenas poblaciones de presas, poco impactados por el ser humano, con poca presión antrópica, con cortados y repisas rocosas y con un paisaje en el que alternan bosques bien conservados junto a espacios abiertos de campiña y pastos. Gran parte de las presas sobre las que preda son apresadas en campos de cultivo de secano, cereal fundamentalmente.

En la zona de estudio han sido constatados 6 núcleos con sendas parejas: Arriano, Karanka, Barron, Hereña y Gatzaga-Buradon, el sexto, se sitúa fuera de la zona de estudio dentro de Angulo, en el nordeste de la provincia de Burgos. A través de estudios previos también se han determinado otros dos territorios con sus parejas en Subijana-Morillas y Aprikano que, sin embargo, a través de esta investigación no han podido ser certificados.

Aparte de las parejas reproductoras, en la época favorable para la reproducción también han sido detectados otros dos individuos solitarios en territorios no ocupados hasta la fecha por ninguna pareja. Uno en las inmediaciones del Santuario de Oro y el otro en Ocio, en las cercanías de la Sierra Portilla. Mientras el primero da la impresión que no es un territorio potencial por la falta de buenos recursos tróficos y mayor presión antrópica, el segundo sí cumpliría con todos los requerimientos de manera que se conforma como una ubicación potencial.

Las densidades poblacionales son realmente elevadas, del orden de 0,48 parejas $/ 100 \mathrm{~km}^{2}, 0,62$ parejas $/ 100 \mathrm{~km}^{2}$ si tenemos en cuenta también las parejas no confirmadas. Si sólo se toman en cuenta los territorios detectados, la zona de estudio se situaría en el $13^{\circ}$ lugar en cuanto a densidad de población de la especie en España. Si tomamos en cuenta también los "supuestos" dos territorios más, la posición sería la $4^{\mathrm{a}}$.

Hasta un $75 \%$ de la población se sitúa en el sector central del territorio, en esa banda bioclimática definida como de transición o submediterránea. El resto se sitúa en territorios más meridionales y claramente mediterráneos. No parece que los sectores más atlánticos o montanos puedan resultar interesantes para la ubicación de nuevas parejas reproductoras.

Aparentemente las grandes infraestructuras (AP-68, A-1 o trazado ferroviario Madrid-Irún) no suponen ningún obstáculo para la ubicación de las parejas de águila real. Tampoco los pequeños núcleos de población con actividades humanas relativamente rutinarias parecen tener una influencia negativa. Sí muestran impactos importantes actividades puntuales como la caza, las labores forestales, la escalada o el montañismo. 
Aunque en los últimos 6 años se ha perdido un territorio, la población de Álava ha crecido entre 1990 y la actualidad en un $63,6 \%$, mostrando 7 territorios nuevos ( 8 en 2010), eso supone un crecimiento bastante considerable. El crecimiento se debe a cuestiones como un mayor grado de protección tanto del taxón como de los hábitats que ocupa, mayor concienciación de la sociedad, el crecimiento de algunas de las poblaciones de especies sobre las que preda y el mayor control administrativo sobre su caza, la utilización de venenos o la expoliación de nidos y pollos.

Aunque el crecimiento haya sido notable en la provincia de Álava, no existen muchas más posibilidades de incrementar nuevos emplazamientos para nuevas parejas en el área de estudio. Es muy probable que la especie se estabilice y se mantenga o se incrementen ligeramente sus contingentes poblacionales a la vista de que existían citas de nidos, parejas o territorios anteriormente, pero que no han sido constatados por nuestra investigación.

Teniendo en cuenta los territorios potenciales que reúnen las características adecuadas y los nidos históricos no detectados en esta investigación, las estimaciones de nuevos territorios y parejas se podrían aproximar a 6. Entre Tuyo y Subijana, en el Monte Toloño, en Valderejo, Monte Gorobel, Valle de Cuartango ( $3^{\mathrm{a}}$ pareja), Sierra de Arcena y la Sierra de Portilla.

Se recomienda continuar con los estudios diacrónicos sobre la especie y sus emplazamientos y parejas de cría para seguir disfrutando de datos poblacionales fidedignos que puedan llevar al control de la misma, de manera que se determine su evolución temporal para, en caso de existir una dinámica negativa, poner en marcha medidas concretas que reviertan sus causas. También parece aconsejable ampliar los estudios introduciendo otras cuestiones referidas a la alimentación y la etología de la especie en este territorio.

\section{Referencias}

Arroyo, B., Ferreiro, E. \& Garza, V., 1990. El Aguila Real (Aquila chrysaetos) en España. Censo, distribución, reproducción y conservación. Colección Técnica. ICONA. Ministerio de Agricultura, Pesca y Alimentación, Madrid.

Arroyo, B., 2000. I Censo regional de Águila Real. Año 2000. Informe inédito para la Consejería de Medio Ambiente. Junta de Castilla y León, Valladolid.

Arroyo, B., 2003. Águila real Aquila chrysaetos. In: Martí, R. y Del Moral, J. C. (Eds.). Atlas de las aves reproductoras de España. Dirección General de Conservación de la Naturaleza y SEO/BirdLife: 188-189 pp., Madrid.

Arroyo, B., 2004. Águila real Aquila chrysaetos. En: Madroño, A., González, C., y Atienza J.C. (Eds.): Libro rojo de las aves de España. Dirección General para la Biodiversidad y SEO/BirdLife: 151-153, Madrid.

Bautista, J., Moleón, M. \& Gil-Sánchez, J.M., 2016. Dieta del Águila Real en el sur de España. Quercus, 364: 16-23.
Bernis, F., 1974. Más sobre fenología de reproducción y status de Aquila chrysaetos en Iberia. Ardeola, 19: 447-454.

Brown, L. \& Amadon, D., 1968. Eagles, Hawks and Falcons of the World, Vol. 1. New York: McGraw Hill.

Collopy, M.W., 1984. Parental Care and Feeding Ecology of Golden Eagle Nestlings. The Auk, 101: 753-760. https://doi. org/10.2307/4086902

Del Moral, J.C., 2009. El águila real en España, Población reproductora en 2008 y método de censo. Madrid: SEOBirdLife.

Díaz, M., Asensio, B. \& Tellería, J.L., 1993. Aves Ibéricas. I. No pasariformes. J.M. Reyero Editor, Madrid.

Eustat, 2016. Datos estadísticos por municipios. Vitoria-Gasteiz: Instituto Vasco de Estadística. Disponible, en marzo de 2020. En: http://www.eustat.eus/municipal/datos_estadisticos/info territorial_c.asp.

Fernández, $\bar{C}$ \& Purroy, J.F., 1990. Tendencias geográficas en la alimentación del Aguila real (Aquila chrysaetos L.) en Navarra. Ardeola, 37 (2): 197-206.

Ibor, S., 2014. El Águila real en Europa. Disponible, en marzo de 2019. En: http://birding140.es/el-aguila-real-en-europa/

Illana A., 1991. El águila real Aquila chrysaetos en Álava. Tasas reproductoras. Informe 1991. Departamento de Agricultura de la Diputación Foral de Álava y Gaden, Vitoria-Gasteiz.

Illana A., 1993. El águila real Aquila chrysaetos en Álava. Tasas reproductoras. Informe 1993. Departamento de Agricultura de la Diputación Foral de Álava y Gaden, Vitoria-Gasteiz.

Illana A., 1994. El águila real Aquila chrysaetos. Tasas reproductoras y situación individualizada de las parejas reproductoras nidificantes en Álava". Informe 1994. Departamento de Agricultura de la Diputación Foral de Álava y Gaden, Vitoria-Gasteiz.

Illana, A. \& Paniagua, D., 1998. El águila real (Aquila chrysaetos). Análisis de la situación actual y Plan Manejo y Gestión de las poblaciones. Diputación Foral de Álava. VitoriaGasteiz. Inédito.

Illana A., Paniagua D., Martínez de Lecea, F. \& Echegaray, J., 2011. El águila real (Aquila chrysaetos) en el País Vasco: Censo, reproducción y caracterización de sus zonas de nidificación 2010. Dpto. de Biodiversidad y participación Ambiental del Gobierno Vasco, Vitoria-Gasteiz. Informe inédito.

Illana A., Paniagua D. \& Martínez de Lecea, F., 2016. El águila real en el País Vasco: Censo, reproducción y caracterización de sus zonas de nidificación 2016. Departamento de Agricultura de la Diputación Foral de Álava y Gaden, Vitoria-Gasteiz.

Lozano, P.J. \& Latasa, I. (Coord.), 2016. El Catálogo de Paisaje de La Llanada Alavesa. Universidad del País Vasco-EuskalHerriko Unibertsitatea, Lejona.

Meaza, G., Lozano, P.J. \& Varela-Ona, R., 2020. Biodiversidad, paisaje y gestión ambiental. Itinerarios biogeográficos por el País Vasco y territorios aledaños. Tirant humanidades, Valencia.

Palomo, L.J. \& Gisbert, J. (Eds.), 2002. Atlas de los mamíferos terrestres de España. Ministerio de Medio Ambiente y SECEM, Madrid.

Pedrini, P. \& Sergio, F., 2002. Regional conservation priorities for a large predator: golden eagles (Aquila chrysaetos) in the Alpine range. Biological Conservation, 103 (2): 163-172.

Uriarte, A., 1996. El Clima de Euskal-Herria. In: Meaza y Ruiz (Eds.). Geografia de Euskal-Herria. Etor argitaletxea, Vitoria-Gasteiz.

Zuberogoitia, I. \& Torres, J.J., 1997. Aves rapaces de Bizkaia. Bilbao Vizcaya Kutxa, Bilbao. 